\title{
Féeries
}

Études sur le conte merveilleux, XVII $-\mathrm{XIX}{ }^{\mathrm{e}}$ siècle

\section{Histoire de la sultane de Perse et des vizirs ; Les Mille et un Jours, contes persans ; Les Aventures d'Abdalla}

François Pétis de la Croix, éd. critique par Raymonde Robert ; Éd. critique sous la dir. de Pierre Brunel avec la collaboration de Christelle Bahier-Porte et de Frédéric Mancier ; Abbé Jean-Paul Bignon, éd. critique par R. Robert, Honoré Champion, Bibliothèque des Génies et des Fées, vol. 8, Sources classiques $n^{\circ}$ 54, Paris, 2006, 1333 p.

\section{Anne Defrance}

\section{OpenEdition}

Journals

Édition électronique

URL : http://journals.openedition.org/feeries/463

DOI : 10.4000/feeries.463

ISSN : $1957-7753$

Éditeur

UGA Éditions/Université Grenoble Alpes

Édition imprimée

Date de publication : 1 octobre 2007

Pagination : 247-255

ISBN : 978-2-84310-101-4

ISSN : 1766-2842

Référence électronique

Anne Defrance, «Histoire de la sultane de Perse et des vizirs ; Les Mille et un Jours, contes persans ; Les Aventures d'Abdalla », Féeries [En ligne], 4 | 2007, mis en ligne le 03 novembre 2008, consulté le 21 septembre 2020. URL : http://journals.openedition.org/feeries/463 ; DOI : https://doi.org/10.4000/ feeries.463

Ce document a été généré automatiquement le 21 septembre 2020.

(c) Féeries 


\section{Histoire de la sultane de Perse et des vizirs ; Les Mille et un Jours, contes persans ; Les Aventures d'Abdalla}

François Pétis de la Croix, éd. critique par Raymonde Robert ; Éd. critique sous la dir. de Pierre Brunel avec la collaboration de Christelle Bahier-Porte et de Frédéric Mancier ; Abbé Jean-Paul Bignon, éd. critique par R. Robert, Honoré Champion, Bibliothèque des Génies et des Fées, vol. 8, Sources classiques $n^{\circ}$ 54, Paris, 2006, 1333 p.

\section{Anne Defrance}

1 Ce volume prend place dans la série consacrée, dans la collection, à la veine des contes orientaux (1704-1789) ${ }^{1}$ inaugurée par la publication des Mille et Une Nuits d'Antoine Galland (1704). Pétis de La Croix traduit et adapte lui aussi des contes, bien davantage remaniés dans son second recueil, Les Mille et Un Jours (1710-1712) que dans le premier, l'Histoire de la sultane de Perse et des vizirs (1707). Quant aux contes de l'abbé Bignon, parus entre 1712 et 1714 et continués, pour l'édition de 1773, par Louis-Daniel Colson, ils sont totalement inventés, Bignon n'étant pas arabisant même s'il dispose d'une grande culture orientaliste. Ainsi, ces trois œuvres témoignent-elles chacune à leur manière des directions prises par la production orientalisante après Galland, explique Raymonde Robert dans sa présentation générale du volume. Directions qui sont donc envisagées ici suivant le degré de fidélité variable des oeuvres par rapport à leurs sources : ce principe fondera d'ailleurs son analyse du premier recueil de Pétis.

2 L'Histoire de la sultane de Perse et des vizirs est présentée par Pétis de La Croix comme la traduction de l'ouvrage de Chéc Zadè (précepteur d'Amurath second), qui nous est inconnu. Le récit-cadre du recueil met en place une sorte de tribunal devant lequel un accusé est défendu par des " avocats », les vizirs conteurs. Il s'agit ici de faire la preuve de la malice des femmes. R. Robert commence par examiner les divers recueils turcs à 7, 10 ou 40 orateurs, recueils construits, comme le texte de Pétis, sur le principe de la rhétorique judiciaire. Il ressort de cette première confrontation que c'est dans l'Histoire des quarante vizirs qu'a puisé Pétis, qui n'a pas repris les histoires en suivant leur ordre 
originel, et les a essentiellement adaptées, même si sa traduction est par endroits fidèle. On sait depuis 1886, date de la traduction en anglais de l'Histoire des quarante vizirs par E.J.W. Gibb, qu'il en existait au XvIII ${ }^{\mathrm{e}}$ siècle au moins 5 versions, toutes différentes. Quoique cette traduction, dont se sert Raymonde Robert pour son édition, recense les variantes entre ces versions, leur confrontation ne permet pas, à ses yeux, de définir avec certitude la version manuscrite dont Pétis s'est servi. Dans les notices qu'elle rédige pour chacun des récits qui composent le recueil de Pétis (récit-cadre et contes insérés), elle compare à chaque fois systématiquement le texte avec la traduction de Gibbs, tout en signalant les concordances avec les types recensés par le Catalogue international Aarne et Thompson. Cette méthode descriptive et comparative débouche ensuite, souvent, sur une interprétation, proposée avec prudence en raison des incertitudes pesant encore sur la version utilisée par Pétis. Sont alors mises en évidence, d'une notice à l'autre, certaines divergences que je classerai ici. Elles tiennent d'abord à la visée essentiellement religieuse du recueil originel (celui de Pétis servirait une intention moralisante), ensuite aux bienséances et aux valeurs du lectorat français de l'époque : la représentation de la femme, par exemple, est loin d'être identique entre les deux civilisations, et Pétis, dans son Histoire du Brahmane Padmanaba et du jeune Fyquaï, modifie la nature de la relation qu'entretiennent ces deux personnages (relation homosexuelle dans la traduction de Gibbs). Ailleurs, c'est la contamination esthétique du conte oriental et des modèles littéraires français qui a pu jouer : ainsi Pétis a-t-il généralement tendance à développer les analyses psychologiques : dans un conte, il a par exemple modifié l'appartenance sociale d'un personnage qui suit un parcours ascensionnel. R. Robert explique cela par le fait que dans le conte de fées littéraire français, on ne rencontre pas de fils de paysan devenant fils de roi (notons quand même que dans Le Maitre Chat ou le Chat Botté de Perrault, le fils d'un meunier devient, à la fin, beau-fils du roi, mais il est vrai que cette situation est exceptionnelle). Enfin, la comparaison avec l'Histoire des quarante vizirs peut faire état d'une prise de position politique. En effet, dans tout le recueil, deux types parfaitement opposés de rois sont confrontés, des bons et des méchants. Dans l'Histoire du vieux roi d'Éthiopie et de ses trois fils, il s'agit pour un bon roi de se choisir un digne successeur. Or Pétis, en développant par rapport au modèle « l'aspect émouvant des relations du roi et de son peuple », a pu désirer produire « un contraste intéressant entre l'image de ce roi, véritable père pour son peuple, et l'attitude réelle du pouvoir en France à l'époque» (p. 44). De telles propositions, toujours prudemment énoncées, fournissent des pistes stimulantes.

Le recueil suivant de Pétis de La Croix, Les Mille et Un Jours, contes persans, a fait l'objet d'un travail éditorial d'équipe. Cette édition critique vient à la suite de l'édition non annotée de Paul Sebag (Christian Bourgois, 1980), revue et complétée en 2003 et parue chez Phébus. La présente édition du texte suit l'originale (1712-1714) en modifiant partiellement sa numérotation des Jours. Les notices qui la précèdent, certaines signées conjointement par P. Brunel et F. Mancier, d'autres par C. Bahier-Porte, tiennent à la fois du résumé et de l'analyse.

Dans sa préface, Pierre Brunel met essentiellement en perspective l'ouvrage de Pétis par rapport au recueil arabe des Mille et Une Nuits découvert presque simultanément, en faisant abondamment référence aux travaux des spécialistes des deux recueils. Il déchiffre dans l'épilogue des Mille et Un Jours le message d'un homme des Lumières: lorsque, le dernier Jour, est vaincue la méfiance d'une princesse à l'égard des hommes (comme l'était, dans la dernière Nuit, celle d'un sultan envers les femmes), Pétis écarte dans le même temps la magicienne Mehrefza et ce dieu Kesaya dont la vision avait 
trompée la princesse, dieu qui « demeurera à jamais caché, à moins que le sanctuaire ne soit vide » (p. 227).

Christelle Bahier-Porte fournit ensuite une longue introduction au recueil de Pétis. Après une biographie très détaillée qui examine de près la relation de l'auteur avec Galland, elle rouvre le « dossier Lesage " pour éclairer la question controversée d'une éventuelle collaboration de cet écrivain au recueil. L'hypothèse à laquelle les spécialistes (sauf P. Sebag) adhèrent, est que sa participation ne se limiterait pas à la simple correction du texte. Une grande quantité d'arguments sont alors convoqués en faveur d'une participation plus étroite de Lesage à la rédaction. On sait qu'il a adapté pour le théâtre de la Foire plusieurs contes des Mille et Un Jours, mais C. Bahier-Porte verse au dossier un nouvel élément renforçant la présomption de la participation du romancier à l'élaboration du recueil : le relevé de nombreux passages du roman Gil Blas de Santillane témoignant au moins de la parfaite connaissance que Lesage avait du recueil et de sa philosophie.

6 Elle analyse ensuite le contenu de l'ouvrage, en fait valoir la relative dimension critique, qui s'exerce surtout dans les domaines de la justice et de la religion, la satire morale y étant présente, mais plus conventionnelle. Elle montre comment dans le recueil s'opère en douceur la mutation du genre du conte oriental vers les voies de la parodie et du récit libertin, dans lesquelles s'engageront plus ostensiblement les conteurs des années 30. À la différence de P. Sebag qui souligne la dimension globalement optimiste du recueil, elle pointe ces quelques contes susceptibles de contredire le projet d'édification morale de l'auteur et d'aller à l'opposé de la démarche thérapeutique du personnage de la nourrice conteuse Sutlulmemé. Elle analyse ensuite la nature dramatique des contes que Lesage adapta sur un mode bouffon pour la Foire, jouant un rôle certain dans le détournement parodique qu'allaient opérer après lui les futurs auteurs de contes. La présentation de l'œuvre s'achève sur le rappel d'une supercherie littéraire souvent commentée : Pétis a avancé masqué en prétendant n'être que le traducteur du derviche Moclès, et l'éditrice renvoie in fine aux sources des contes repérées en leur temps par P. Sebag.

7 Frédéric Mancier ajoute à la préface et à l'introduction précédentes une étude littéraire $\mathrm{du}$ recueil de Pétis. Après avoir contesté, sans malheureusement apporter aucun argument, la participation de Lesage au recueil, hypothèse soutenue précédemment par C. Bahier-Porte, il montre comment le recueil servirait - relativement - «une esthétique de la quantité ». À ses yeux, la conteuse nourrice des Jours ne vaut pas Schéhérazade, car il lui «manquera toujours la poésie» (p. 278). Autre affirmation que l'on peut trouver un peu abrupte: Pétis «ne sait qu'inscrire des noms» (p. 279), d'ailleurs il ne "s'embarrasse pas [dans le premier conte] d'évocations créatrices d'espaces et de mirages» (p. 278). L'auteur de l'étude voit dans le recueil une logique qui «ne doit pas différer de ce qu'on peut appeler la poétique élitiste du conte » (p. 280) : on aurait aimé quelques éclaircissements sur la formule, ils seront peut-être partiellement fournis à la fin de l'étude à travers l'évocation du lectorat mondain visé par Pétis. Mais peut-on pour autant attribuer au genre tout entier une telle poétique? En outre, F. Mancier fournit une explication, qui ne me parait pas totalement convaincante, du non-respect par l'auteur de la promesse du titre : le contrat liminaire des mille et un jours ne serait pas rempli parce que «le processus de transformation intérieure [de la princesse Farrukhnaz est] véritablement engagé » et qu'elle est « apaisée, convertie, appelée au bonheur dans le Jour Mille et un et dernier » tandis que 
«sa personnalité [est] devenue plus attachante» (p. 280). Peut-être, mais pourquoi alors avoir coupé précisément entre le jour $\mathrm{CXC}^{\mathrm{e}}$ et le jour $\mathrm{CMLX}^{\mathrm{e}}$, pourquoi n'avoir pas mis un terme au recueil avant d'être parvenu au nombre fatidique, est en droit de se demander le lecteur. L'auteur de l'essai voit seulement dans ce raccourci une liberté prise à l'égard de «l'esthétique de la quantité ». Il évoque ensuite la virtuosité de la composition du recueil qui ne se contente par de juxtaposer les histoires (structure dominante quand elles sont contées par la nourrice), recueil qui suit globalement une progression démonstrative et par moments utilise la technique complexe de l'encastrement, créatrice de surprise et « d'effets de couleur » (284). F. Mancier repère aussi dans les Mille et Un Jours un cycle de Bedreddin-Lolo, comme on a pu parler d'un cycle de Harroun-Arraschid dans les Mille et Une Nuits. Ce cycle est dominé par une vision pessimiste du monde, des voix convergentes y répétant l'impossibilité d'être heureux. L'étude s'achève par celle du merveilleux dans le recueil, un merveilleux qui, tout en étant oriental, situe ses métamorphoses dans des registres propres à Pétis. Même si l'un des personnages, Symorgue, vainqueur de la magicienne, en vient, au terme de son itinéraire, au service de Dieu, le chrétien Pétis n'a pas fait d'un recueil visant le public mondain un livre mystique, estime l'auteur de l'essai, non sans avoir défini que « Simorg » est symbole de Dieu et de la quête de l'absolu. La préface de Pierre Brunel, on le rappelle, s'achevait sur la mention d'un dieu caché (Kesaya), et l'hypothèse formulée d'un sanctuaire vide, symptôme révélateur de l'esprit des Lumières. Les conclusions de ces deux analyses du merveilleux dans le recueil, mises au compte d'une idéologie plus ou moins latente, étant, on le sent bien, divergentes en dépit de leur possible point de contact, un lecteur qui serait en quête d'une idée plus nette de la fonction du merveilleux et de la religion dans l'œuvre, ou de la position de Pétis sur la question, se trouve finalement renvoyé au texte. On ne peut évidemment pas totalement le regretter...

8 Venons en enfin au dernier recueil édité dans ce volume par Raymonde Robert, Les Aventures d'Abdalla de l'abbé Jean-Paul Bignon (1712-1714). Si l'ouvrage a connu une véritable fortune au $\mathrm{XVIII}^{\mathrm{e}}$ siècle, ce dont témoignent ses rééditions, il est tombé dans l'oubli par la suite. De ce fait cette première édition moderne défriche des terres quasi inexplorées par la critique. L'édition de référence est l'édition originale de 1712-1714 et R. Robert utilise aussi celle de 1773, comme l'éditeur du Cabinet des fées en 1785, pour la continuation de Colson. Seront signalées les variantes entre l'édition princeps et l'édition de 1773. Précisons pour notre part que cette continuation ne fut pas la seule: en janvier 1778 la Bibliothèque des romans en offre une autre, qui pourrait être de la plume de M. de Paulmy, selon Barbier.

9 Raymonde Robert $a$, dans ce volume, donné la primeur à la synthèse plutôt qu'aux analyses de détail, préférant grouper ses remarques dans une riche introduction plutôt que de les égrener dans des notices, récit par récit. À ses yeux, le recueil de Bignon tranche sur la production des conteurs, arabisants ou non, entraînés par la déferlante orientalisante, tant par la précision de la documentation, la qualité, la variété et le caractère inattendu de ses situations que par l'étonnante ouverture d'esprit de son auteur, grand dignitaire de l'église catholique. Ainsi l'œuvre, à ses yeux, se placerait à cet égard loin devant Les Mille et Une Nuits de Galland. Bignon, visiblement épris d'une intention didactique, "loin de chercher à atténuer les différences de mœurs, de religions ou de coutume, [...] les souligne de toutes les façons possibles ", au point d'offrir au lecteur " une sorte de guide touristique qui lui permet de comprendre et d'imaginer ces pays si lointains et si différents » (p. 913). L'oeuvre est originale aussi 
par le fait que, contrairement aux Nuits, les aventures des personnages, pour imaginaires qu'elles soient, sont très précisément contextualisées, situées géographiquement et datées, et qu'elles font état de connaissances géographiques et historiques très pointues. Bignon met donc à profit, outre la lecture de la Bibliothèque orientale de d'Herbelot et du Coran, celle de nombreux récits de voyage auxquels il a eu un accès privilégié comme directeur de la librairie et chef de la censure jusqu'en 1714 (il deviendra quelques années plus tard bibliothécaire du roi).

10 Mais les connaissances de Bignon ne se limitent pas à la civilisation orientale : son recueil témoigne aussi de sa culture dans le domaine du folklore français, remarque R. Robert. C'est ainsi que dans l'un des récits encastrés, l'Histoire de la princesse Zeineb et $d u$ roi Léopard, les folkloristes ont reconnu l'une des versions du conte-type très représenté dans le domaine français et notamment par les d'Aulnoy, Murat, etc., tout comme, plus tard, par $\mathrm{M}^{\mathrm{me}}$ de Villeneuve (La Belle et la Bête). L'éditrice note également la présence, sous un travestissement à l'orientale, de certains motifs empruntés au folklore et à la littérature française. Ils sont recensés dans l'introduction, et des notes infrapaginales y renverront rapidement au fil du texte, conformément à une volonté exprimée de ne pas l'alourdir.

11 Raymonde Robert s'intéresse ensuite à l'usage que fait Bignon d'un merveilleux qui est loin d'être chez lui purement ornemental. Ses positions sont clairement exprimées dans deux textes introducteurs, l'Avertissement du recueil et la lettre à Monsieur Sandisson au traducteur. Dans ces textes théoriques, l'auteur opère le classement entre un bon surnaturel, inoffensif voire utile parce qu'il peut aider à vivre dans la vertu et mener à la connaissance du vrai Dieu, et un mauvais, qui mène au péché. Par ailleurs, l'auteur y fait le procès de la littérature féerique des années précédentes, et réhabilitant le merveilleux médiéval, les chevaliers chrétiens ou guerriers légendaires orientaux. R. Robert explique l'apparente contradiction qui consiste, chez l'auteur, à faire usage lui-même d'un surnaturel délirant: c'est son intention louable qui le justifie et l'autorise. Il a choisi, dans le recueil, de prêter sa voix à un musulman, Abdalla, doté d'un esprit de tolérance remarquable, quoique ce personnage s'élève contre les religions polythéistes ou idolâtres: cette orientation met en valeur les points de convergence entre les religions catholique et musulmane. Les analyses doctrinales présentées ici, nourries d'intéressantes références à l'actualité des questions religieuses contemporaines, permettent de lever l'ambiguïté du recueil dans ce domaine. Si Bignon, par son éducation, est proche des Jansénistes, l'esprit d'ouverture et le syncrétisme dont il fait preuve tout au long de son ouvrage rappelle l'attitude des Jésuites missionnaires. Or cette attitude avait été finalement condamnée en 1715 par le Pape, ce qui pourrait en effet expliquer ces quelques notes infrapaginales d'auteur, notes peu aimables à l'encontre de la religion musulmane. Ce serait pour lui une manière, affichée dans les marges du récit, d'atténuer ou de dissimuler son déviationnisme.

12 L'édition des Aventures d'Abdalla offre également l'intérêt de présenter la reproduction de la suite gravée des 8 figures en taille douce qui ornent l'édition originale, figures fort peu connues. L'édition de 1745 des Aventures d'Abdalla, imprimée chez Damonneville (futur éditeur des Voyages de Gulliver traduits par Desfontaines) ajoute 4 autres illustrations aux 8 originales, et elles sont réalisées dans une manière similaire. Cette édition, la $3^{\text {e }}$ de l'œuvre, est signalée par le catalogue de la BNF, mais oubliée par R. Robert dans ses deux recensements p. 909 et 1310. Les fiches de la BNF relatives aux 
deux éditions posthumes de 1745 et 1773 mentionnent Bignon non seulement comme auteur, mais comme illustrateur. La question mériterait donc d'être éclaircie. Les illustrations d'une première édition sont très rares au XVIII ${ }^{e}$ siècle et elles sont, qui plus est, exceptionnelles dans les recueils de contes: je serais tentée de voir dans la publication de cette luxueuse édition, très coûteuse à sa parution, n'en doutons pas, une opération de prestige et une volonté de surenchère sur les autres recueils, orientaux ou non. Ceux qui s'intéressent à l'illustration des textes du XviII siècle pourront remarquer la particularité de celle-ci, qui met l'accent sur un merveilleux spectaculaire, ce qui ne sera pas l'option retenue par Marillier, illustrateur de tous les volumes du Cabinet des fées. Dans le volume consacré aux contes de Bignon qui paraîtra en 1785 , il se montrera, quant à lui, bien davantage intéressé par l'expression de la couleur locale (costumes, décors orientaux), ou par la représentation de la violence (un sabre levé sur un personnage, par exemple, scène récurrente qu'il représente dans plusieurs recueils, et qui l'avait déjà été un siècle plus tôt dans le recueil des Histoires ou contes du temps passé de Perrault, pour sa Barbe Bleue ${ }^{2}$ ). L'illustrateur de l'édition originale de Bignon n'a dédaigné ni l'attrait pour l'exotisme, ni le potentiel émotionnel d'une scène digne de faire ressurgir des peurs primaires, mais il y associe une question plus sensible ces années-là, qui est celle du merveilleux. Toutes les illustrations montrent des merveilles, en effet (il n'en manquait pas, dans cette édition qui les fait se succéder à vitesse surprenante). Or, à l'époque, la querelle entre les Anciens et les Modernes connaissait une nouvelle flambée : la polémique fait à nouveau rage à partir de la traduction d'Homère par $\mathrm{M}^{\text {me }}$ Dacier, en 1711, à laquelle répondra bientôt Houdar de la Motte. La querelle du merveilleux chrétien et du merveilleux païen qui avait agité les esprits depuis la deuxième moitié $d u x$ VII $^{e}$ siècle est donc à nouveau ravivée, avec celle du mensonge de la Fable. Le texte de Bignon, parfaitement contemporain de la mêlée, au moment où Fontenelle prépare son ouvrage sur l'Origine des fables, y ajoute un écho personnel. Bignon est directeur du comité d'hommes de lettres qui préside de 1701 à 1714 le Journal des Savants. Ce périodique se fait l'écho de la querelle, dans les années de la publication des Aventures d'Abdalla, sans prendre parti mais en jouant les arbitres. En exhibant dans ce recueil, de manière totalement immédiate, la sensationnelle illustration et, en place d'honneur, un spectaculaire frontispice qui ne connote pas l'Orient contrairement aux figures suivantes, Bignon nous montre que son merveilleux est non seulement assumé, mais revendiqué. Cela ne va pas sans paradoxe et sans ironie, peut-être, puisqu'on charge ici une nouvelle fable de dénoncer les mensonges de l'occultisme, et qu'on remet à une péris (équivalent, en Orient, d'une bonne fée) le soin de condamner ses consœurs françaises, qualifiées ici de «fées chimériques ». Le frontispice des Aventures d'Abdalla reprend la scène du jugement du comte de Gabalis racontée dans la Lettre de Monsieur Sandisson au traducteur. On y voit un Occidental terrifié, en passe d'être dévoré par un gigantesque monstre, gueule béante, au premier plan, face à une assemblée dirigée par la reine des péris. Représentation tout à fait intéressante, ce me semble, puisqu'elle focalise tout le recueil sur la guerre livrée contre l'imposture des sciences occultes avant même de magnifier l'exotisme oriental (il est même assez curieux de voir que la péris est représentée dans le costume classique d'une fée à la française, avec grand chapeau pointu surmonté d'un voile, suivant certaines illustrations du XVIII ${ }^{\mathrm{e}}$ siècle et selon l'imagerie populaire telle que l'a reprise de nombreux illustrateurs de contes pour enfants par la suite). Dans la même lettre, de nombreuses références aux personnages de Madame de Murat, relevées par Raymonde Robert, les accusent pour leur niaiserie, en reprenant une partie des 
arguments développés dix ans plus tôt par les adversaires du genre. Il est d'ailleurs piquant d'entendre les femmes auteurs de contes de fées, pour punition de leurs divagations, condamnées par la première reine des péris à être des "malpropres": telle était justement, rappelons-le, l'une des caractéristiques attribuées par $\mathrm{M}^{\mathrm{me}} \mathrm{de}$ Murat aux anciennes fées françaises (entendre ici les nourrices du folklore ainsi que leurs créatures), dont cette conteuse entendait précisément se dissocier dans son Épitre aux fées modernes (Histoires sublimes et allégoriques, 1699), faisant l'éloge de la production féerique moderne et féminine. La même reine décide, de plus, de soumettre les ouvrages des futures conteuses à la censure de leurs confrères masculins, pour qu'ils en retranchent « les contradictions, les exagérations, les redites» de leurs ouvrages, mais enjoint seulement les conteurs "d'interroger des femmes habiles et ingénieuses, lorsqu'il s'agira de sentiments et d'ajustements » (p. 951). Bignon se situe bien ici aux antipodes d'un Mailly ${ }^{3}$, et se démarque ostensiblement des positions féministes défendues par Perrault et les Modernes une dizaine d'années plus tôt. La comparaison de son Histoire de la princesse Zeineb et du roi Léopard avec les versions féminines du même conte-type est, à cet égard, très révélatrice ${ }^{4}$.

Ce volume, riche de la diversité des points de vue et des méthodes qui s'y complètent et, sur quelques points, s'y opposent, présente enfin, conformément aux principes de la collection, une bibliographie éditoriale, une bibliographie critique, des notices analytiques, les résumés des différentes histoires les composant, et un index des personnages toutes œuvres confondues, où sont mentionnés les titres des histoires, non des recueils, ainsi que la référence de leur première apparition.

\section{NOTES}

1.9 volumes sur les 20 prévus dans la collection sont actuellement publiés, en comptant le volume n 5, De la comédie à la critique - 1690-1709 (éd. Nathalie Rizzoni et Julie Boch), dont la sortie est annoncée pour le 30 mars 2007.

2.Sur la question, voir Christophe Martin, « Dangereux suppléments », l'illustration du roman en France au dix-huitième siècle, Éditions Peeters, La République des Lettres 24, Louvain-Paris, 2005 et, du même auteur, «L'illustration du conte de fées (1697-1789) », CAIEF n ${ }^{\circ}$ 57, mai 2005, p. 113-132.

3.Voir l'introduction aux contes de cet auteur par Tony Gheerhaert, B.G.F. $n^{\circ}$, Champion, 2005.

4.Je me permets de renvoyer ici à mon article, « Figures de Psyché dans quelques contes de fées des XVII et XVIII ${ }^{\mathrm{e}}$ siècles ", actes du colloque Les Métamorphoses de Psyché, Université de Valenciennes, 4-5 décembre 2003, édités par Carine Barbafiéri et Chris Rauseo, C.A.M.E.L.I.A., Presses universitaires de Valenciennes, 2004, p. 171-182. 